\title{
Reducing iron accumulation: A potential approach for the prevention and treatment of postmenopausal osteoporosis (Review)
}

\author{
BIN CHEN $^{1}$, GUANG-FEI LI ${ }^{1}$, YING SHEN ${ }^{1}$, XI HUANG $^{2}$ and YOU-JIA XU ${ }^{1}$ \\ ${ }^{1}$ Department of Orthopedics, The Second Affiliated Hospital of Soochow University, Suzhou, Jiangsu 215004, \\ P.R. China; ${ }^{2}$ Division of Rheumatology, NYU Hospital for Joint Diseases, New York, NY 10003, USA
}

Received August 5, 2014; Accepted April 27, 2015

DOI: $10.3892 / \mathrm{etm} .2015 .2484$

\begin{abstract}
Postmenopausal osteoporosis (PMOP) is a systemic bone metabolism disease, characterized by progressive bone loss following menopause and a subsequent increase in fracture risk. Estrogen deficiency as a result of menopause is known to increase bone resorption and accelerate bone loss. Furthermore, postmenopausal women may exhibit iron accumulation, in addition to estrogen deficiency. Elevated iron levels are a risk factor for PMOP in postmenopausal women, and reducing the iron overload has been demonstrated to benefit bone cell metabolism in vitro and improve the bone in vivo by normalizing osteoclastic bone resorption and formation. The identification of hepcidin was a key development in the field of iron metabolism in the previous decade. We hypothesize that hepcidin may aid in the prevention and treatment of PMOP due to its capacity to control body iron stores and its intrinsic effects on osteoblast function. The aim of the current review was to highlight the role of iron accumulation in the pathogenesis of PMOP and to evaluate the possible use of hepcidin as a potential therapy for this condition.
\end{abstract}

\section{Contents}

1. Introduction

2. Iron accumulation in postmenopausal women

3. Involvement of estrogen in iron homeostasis

4. Iron overload and abnormal bone metabolism

5. Reducing iron overload for the prevention of bone loss

6. Hepcidin treatment: A potential approach for the reduction of iron overload

7. Future prospects

Correspondence to: Professor You-Jia Xu, Department of Orthopedics, The Second Affiliated Hospital of Soochow University, 1,055 Sanxiang Road, Suzhou, Jiangsu 215004, P.R. China

E-mail: xuyoujia@medmail.com.cn

Key words: iron, osteoporosis, hepcidin, postmenopause

\section{Introduction}

Due to the progressive aging of populations worldwide, osteoporosis is a growing public health concern, with increasing prevalence among aging individuals, particularly postmenopausal women. Although osteoporosis has been recognized as a disease entity for almost a century, therapeutic approaches are limited, since the pathogenesis of postmenopausal osteoporosis (PMOP) is complex and not yet fully elucidated. Thus, recent progress towards understanding the role of iron accumulation in PMOP is crucial, since it may expose the underlying mechanisms and aid the treatment of this bone disease.

Iron is one of the most abundant transition metals in the human body, and serves a key function in numerous biological processes, including oxygen transport, DNA synthesis and energy production (1). However, excessive iron is deleterious to organ function (2). If the iron concentration in the circulation exceeds the binding capacity of transferrin, an iron-binding blood plasma glycoprotein, then free iron or non-transferrin-bound iron becomes abnormally enriched in various organs, including the liver, heart, brain and pancreas (3). As a consequence, organs are subject to potentially irreversible damage. Previously, Weinberg $(4,5)$ hypothesized that iron overload is a risk factor for osteoporosis. In women, the levels of iron in the form of ferritin (an iron storage protein) have been observed to increase markedly following menopause (6). Furthermore, previous studies have indicated that increasing iron concentrations contribute towards the development of PMOP by enhancing bone resorption and suppressing bone formation, a mode of action which is independent from that of estrogen $(7,8)$. A reduction in iron levels, using either hepcidin (a negative regulator of iron absorption) or an iron chelator, targets the underlying cause and may provide a viable therapeutic option for mitigating the iron accumulation associated with PMOP. The aim of the present review was to investigate the role of iron accumulation in the development of PMOP and to evaluate the use of iron mitigation as a potential therapy for this clinical condition.

\section{Iron accumulation in postmenopausal women}

Iron overload is defined as the presence of high serum ferritin concentrations of $\geq 300 \mu \mathrm{g} / \mathrm{l}$ in men and $\geq 200 \mu \mathrm{g} / \mathrm{l}$ in 
women (9). In recent years, an increasing number of studies have investigated the associations among ferritin, estrogen and PMOP, in order to determine the reason for the enhanced risk of developing osteoporosis in women compared with men. By compiling studies on the levels of ferritin and sex hormones in various populations, it was concluded that as women age, their serum levels of estrogen decrease, while serum ferritin levels increase (10). These results demonstrated a negative correlation between ferritin and estrogen levels during the menopausal transition period (Fig. 1A) (6). With regard to the changes in ferritin and testosterone levels in men, a synchronized pattern was observed as the men age, in which ferritin levels decreased gradually following 'andropause' (Fig. 1B) (11). However, serum ferritin levels in women and men did not reach levels defined as iron overload. Collectively, these results indicated that iron accumulation was a common process in aging women, but not iron overload, which may account for the observed differences between genders in the incidence of osteoporosis. Our retrospective study indicated that women aged $>70$ years with a hip fracture possessed higher serum ferritin levels and significantly reduced bone mineral density (BMD) in the lumbar spine and hip, as compared with a control group (12). In order to eliminate the possibility that osteoporosis itself, but not iron accumulation, exerted an effect on bone metabolism, a team of scientists in Seoul conducted a three-year longitudinal health promotion center-based study on 1,729 subjects, which included 789 middle-aged men and 940 postmenopausal women (13). Subjects with illnesses known to affect ferritin levels or bone metabolism, such as inflammatory diseases, chronic liver diseases or a history of transfusion, were excluded from the study. The results revealed a linear association between vertebral fracture prevalence and serum ferritin levels in women; however, this correlation was not observed in the male subjects (14), which partially supported the previous observations (12). In addition, previous studies have demonstrated that in healthy individuals, increased serum ferritin levels were associated with an accelerated rate of bone loss, which was most marked in women aged $>45$ years $(13,14)$. Notably, levels of serum ferritin were markedly increased in the women aged $>45$ years, as is shown in Fig. 1A. Assuming these results were not a coincidence, 45 years of age, typically during the perimenopausal period, appears to be a critical time point at which the routine examination of biological markers of iron levels may be advisable, in order to monitor the development of iron accumulation.

\section{Involvement of estrogen in iron homeostasis}

On the basis of the aforementioned clinical results, an investigation into the interaction between estrogen and iron levels was conducted. Menstruation is a key process in women of a reproductive age, which is characterized by periodic fluctuations in estrogen and the discharge of blood. For menstruating women, the excretion of endogenous iron occurs primarily through blood loss, resulting in reduced levels of ferritin and an increased prevalence of iron deficiency $(15,16)$. Following menopause, iron is no longer lost through menstruation, and the metal ion increasingly accumulates in the body. However, the interaction between estrogen and iron is not exclusively a result of the effects of estrogen on menstrual blood flow.
Through investigating the effect of estrogen on hepcidin, a negative regulator of iron absorption, estrogen was observed to transcriptionally suppress the expression of hepcidin by binding to the estrogen response element in the hepcidin promoter $(17,18)$. Notably, this process provides a compensatory mechanism through which estrogen prevents the rapid reduction in body iron in menstruating women, in addition to mitigating the accumulation of iron in postmenopausal women.

\section{Iron overload and abnormal bone metabolism}

A number of experimental models of iron overload have been established in vivo in order to confirm the adverse effect of iron on bone metabolism. Tsay et al (7) generated a group of iron-overloaded mice via injection with iron dextran for two months. The results indicated that the iron-overloaded mice exhibited alterations in bone microarchitecture, including the trabecular number, thickness and bone volume fraction, in addition to an increase in bone resorption, as compared with the control group. Similarly, postmenopausal rats fed an iron lactate diet for 4 weeks exhibited a significant increase in urinary deoxypyridinoline, indicating an increase in bone resorption activity (19). In an additional study, pigs were administered $300 \mathrm{mg}$ iron dextran per day intramuscularly for 36 days, after which the pigs appeared to have accumulated large iron deposits in the osteoblasts and bone matrix. Furthermore, the bone mineralization and formation in the pigs were shown to have significantly decreased (8).

The mechanisms underlying the impact of iron on bone metabolism are yet to be fully elucidated. However, in vitro data indicated that iron-induced bone damage was predominantly attributable to the function of iron in catalyzing the formation of reactive oxygen species (ROS) via the Fenton reaction (20). Wnt signaling is essential for bone formation through the stimulation of osteoblastogenesis (21). However, ROS are able to antagonize Wnt signaling in osteoblast precursors by diverting $\beta$-catenin from $\mathrm{T}$ cell factor to Forkhead Box O-mediated transcription, thereby attenuating bone formation (22). Furthermore, a previous study indicated that ferric ion promotes the differentiation of osteoclasts and increases bone resorption via the generation of ROS (23). In summary, the risk of osteoporosis is increased through the suppression of bone formation and enhancing bone resorption.

\section{Reducing iron overload for the prevention of bone loss}

A previous study reported that iron overload was associated with osteoporosis in ovariectomized (OVX) rats (24). When the OVX rats were fed orally with a bone-targeted chelator (1-N-Docosyl-triethylenetetramine pentaacetic acid), bone loss was alleviated significantly in the chelator-treated OVX rats when compared with the untreated-OVX controls $(24,25)$. Desferrioxamine (DFO), an iron chelator isolated from Streptomyces pilosus, is currently used in clinical practice for the treatment of iron overload in patients with thalassemia, hemochromatosis and sickle cell anemia (26-28). Experimental results have indicated that DFO is able to inhibit osteoclastic differentiation, which has been associated with reduced mitochondrial biogenesis 

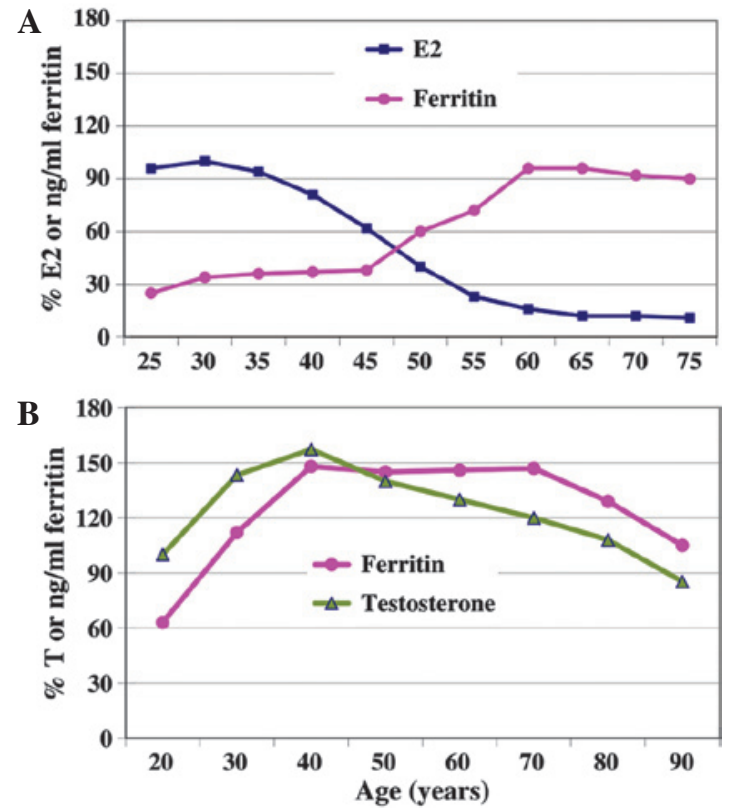

Figure 1. Alterations in the levels of ferritin, E2 and testosterone in women and men over time. (A) Serum levels of E2 were converted to a percentage of the normal value in the serum of 25 -year-old women $(500 \mathrm{pg} / \mathrm{ml})$. (B) Serum levels of testosterone were converted to a percentage of the normal value in the serum of 20-year-old men $(4.4 \mathrm{ng} / \mathrm{ml})$. Levels of ferritin are expressed as $\mathrm{ng} / \mathrm{ml}$ serum. E2, 17ß-estradiol; T, testosterone.

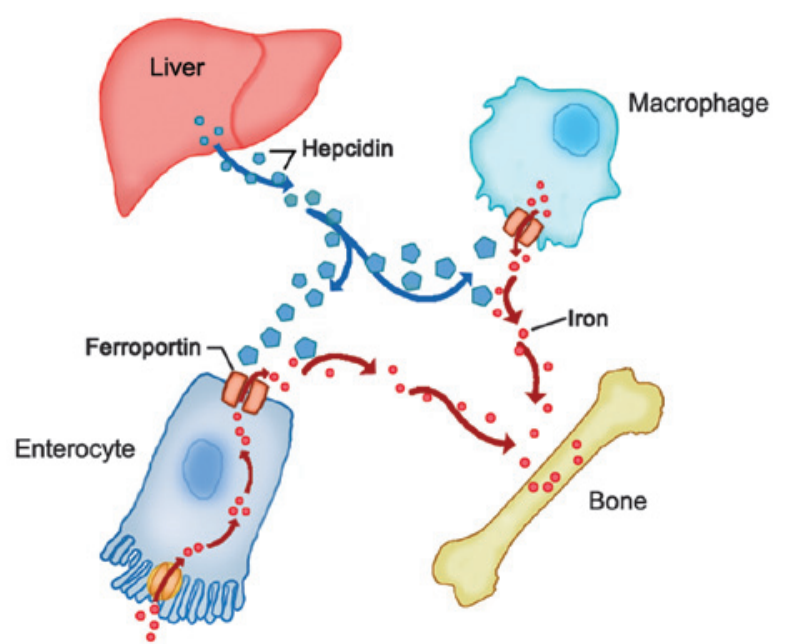

Figure 2. Hepcidin-ferroportin interaction controls the entry of iron into bone tissues. The rate of iron entry into the bone tissues depends primarily on the serum levels of hepcidin. When serum hepcidin levels are reduced, the ferroportin activity is not blocked effectively. Thus, the release of iron from enterocytes and macrophages increases, resulting in elevated serum iron levels and increased iron deposition in bone tissues.

and the production of ROS (29). Furthermore, OVX rats treated with DFO have been shown to exhibit reduced bone resorption and an improved three-dimensional bone structure (29). In addition, our unpublished preliminary data indicated that OVX rats intraperitoneally treated with DFO for three months presented with significantly increased BMD values, accompanied with reduced serum ferritin levels. On the basis of the knowledge that menopause results in iron accumulation, which may independently increase the risk of osteoporosis, the manipulation of iron levels using an iron

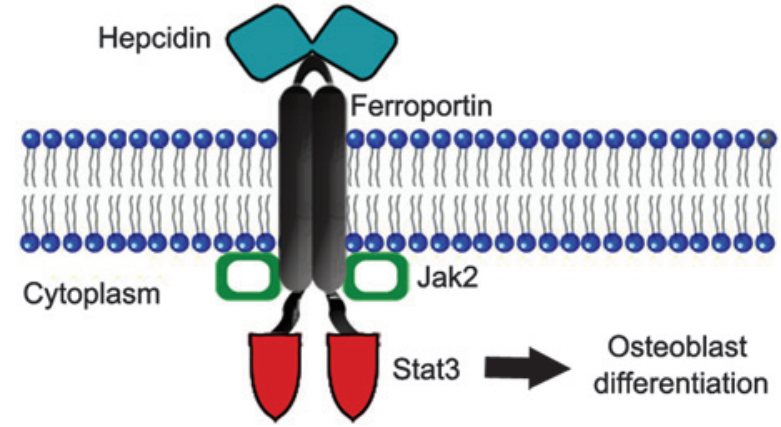

Figure 3. Mechanism through which hepcidin stimulates osteoblast differentiation. The binding of hepcidin to ferroportin activates the Jak2 protein kinase, which subsequently results in osteoblast differentiation mediated by the Stat 3 transcription factor. Jak2, Janus kinase 2; Stat3, signal transducer and activator of transcription 3 .

chelator is hypothesized to be a viable therapeutic approach for the treatment of PMOP.

\section{Hepcidin treatment: A potential approach for the reduction of iron overload}

Iron homeostasis is closely regulated at the point of iron absorption and storage. Hepcidin, a peptide hormone produced by the liver, is the master regulator of iron homeostasis (30). Hepcidin functions by inhibiting the efflux of cellular iron into the circulation through the transmembrane protein receptor, ferroportin. To date, ferroportin is the only known cellular iron exporter in vertebrates, and is known to be highly expressed in cells involved with iron handling, such as duodenal enterocytes, which absorb iron from the diet, and splenic macrophages, which recycle iron from senescent erythrocytes (31). Hepcidin binds to ferroportin on the surface of duodenal enterocytes and splenic macrophages, and induces the internalization and lysosomal degradation of ferroportin, thereby reducing the body's iron stores and iron deposition in the bone (Fig. 2) (32). Therefore, if the hepcidin-induced downregulation of ferroportin is inadequate or ineffective, ferroportin activity is upregulated and iron overload may occur (33-35).

The Hfe gene encodes a membrane protein that is implicated in the stimulation of hepcidin expression (37). In a previous study using $\mathrm{Hfe}^{-/-}$mice, the trabeculae surface was found to be markedly labeled with Prussian blue (used for detecting ferric iron), indicating a considerable quantity of iron deposition in the skeletal tissues. In addition, the $\mathrm{Hfe}^{-/-}$mice manifested an osteoporotic phenotype characterized by low bone mass and impaired bone microarchitecture, in addition to an increased number of osteoclasts along the trabeculae surfaces (38). The results suggested that hepcidin deficiency increases the bone iron content and reduces the quantity of bone tissue. Furthermore, constitutive activation of hepcidin expression or treatment with synthetic hepcidin has been demonstrated to prevent iron overload and the corresponding complications in $\mathrm{Hfe}^{-/}$mice $(39,40)$. In mice with $\beta$-thalassemia, increasing hepcidin expression was shown to induce a reduction in iron content and an improvement in anemia (41). Collectively, these results indicate that hepcidin may possess therapeutic potential for iron-overload diseases (42-44). 
In a rat model of osteoporosis, liver hepcidin gene expression was observed to reduce over time, which further suggested that the development of osteoporosis was associated with reduced levels of hepcidin (45). Furthermore, increased mineralization and reduced rates of apoptosis were observed in human osteoblasts treated with hepcidin (46). In addition, a previous study observed that hepcidin was able to increase the intracellular calcium concentration in cultured osteoblasts, an effect that was more evident in cells growing in a high iron concentration environment (47). By reducing the calcium influx from extracellular spaces using nimodipine (a specific L-type $\mathrm{Ca}^{2+}$ channel blocker) or EDTA (an extracellular calcium chelator), hepcidin-mediated calcium inflow was found to occur predominantly via L-type $\mathrm{Ca}^{2+}$ channels (48). Furthermore, the intracellular calcium induced by hepcidin was sourced primarily from the endoplasmic reticulum, which is triggered by calcium influx (47). Thus, increased levels of intracellular calcium may be associated with the anti-osteoporosis effect of hepcidin. Furthermore, considering that postmenopausal women exhibit enhanced iron accumulation, we hypothesize that hepcidin may provide a viable therapeutic option for the prevention and treatment of PMOP by reducing the iron content in the body and enhancing osteoblast mineralization. In recent years, a patent was filed in the USA detailing the treatment of osteoporosis with hepcidin in perimenopausal and postmenopausal women (49). However, further studies are required to validate this hypothesis.

Notably, there is a potential receptor-based mechanism through which hepcidin may interact with osteoblasts. Previous studies have indicated that the mechanism underlying hepcidin-mediated internalization of ferroportin may result from the activation of the Janus kinase 2/signal transducer and activator of transcription 3 (JAK2/STAT3) signaling pathway $(50,51)$. Furthermore, activation of the JAK2/STAT3 pathway has been reported to promote osteoblast differentiation $(52,53)$, while inhibition of the JAK2/STAT3 pathway using the JAK2 inhibitor, AG490, has been shown to reduce human osteoblast differentiation and mineralization (54). Recently, our research group recognized that ferroportin can be detected in human hFOB 1.19 cultured cells, which indicates that osteoblasts are a potential target of hepcidin activity (55). Based on these collective results, a possible mechanism through which hepcidin stimulates osteoblast differentiation was proposed (Fig. 3).

\section{Future prospects}

In the previous decade, research into iron metabolism and bone metabolism has progressed rapidly; the results of which have improved the understanding of the pathogenesis underlying PMOP (44). The maintenance of iron homeostasis in postmenopausal women has been recognized as crucial, and indicates the therapeutic potential of the manipulation of iron levels for treating PMOP. An artificial, biologically active form of hepcidin, known as 'minihepcidin', has been developed by Preza et al (56).

The following are the key recommendations for clinical research and practice, based on the present review. Firstly, well-designed prospective studies are required to investigate whether DFO or other iron chelators are able to mitigate bone loss in patients with iron overload conditions, such as thalas- semia, hemachromatosis and sickle cell anemia. Secondly, the symptoms of iron overload are insensitive and nonspecific, which differs from the activity of other biologically important metal ions, such as potassium. Therefore, routine examination of the biochemical markers of iron stores may be advisable in order to predict the future patient risk of PMOP. As aforementioned, the optimum age for initiating iron store examination in an aging population is $\sim 45$ years. Thirdly, the epidemiological profile of iron deficiency remains among the most prevalent micronutrient deficiencies worldwide, increasing the risk of diminished bone metabolism in animals and humans $(57,58)$. Therefore, the maintenance of normal iron levels is essential in clinical practice for healthy bone homeostasis.

\section{Acknowledgements}

The authors thank Dr Yi-Lin Yan and Dr Han Wang for critically reading the manuscript. The study was supported by grants from the National Natural Science Foundation of China (nos. 81273090 and 81302438), the Special Program for Clinic of Jiangsu Province (no. BL2014044) and the Natural Science Foundation of Soochow University (no. SDY2013A33).

\section{References}

1. MacKenzie EL, Iwasaki $\mathrm{K}$ and Tsuji Y: Intracellular iron transport and storage: From molecular mechanisms to health implications. Antioxid Redox Signal 10: 997-1030, 2008.

2. Olivieri NF, Liu PP, Sher GD, et al: Brief report: Combined liver and heart transplantation for end-stage iron-induced organ failure in an adult with homozygous beta-thalassemia. N Engl J Med 330: 1125-1127, 1994.

3. Kohgo Y, Ikuta K, Ohtake T, Torimoto Y and Kato J: Body iron metabolism and pathophysiology of iron overload. Int J Hematol 88: 7-15, 2008.

4. Weinberg ED: Iron loading: A risk factor for osteoporosis. Biometals 19: 633-635, 2006.

5. Weinberg ED: Role of iron in osteoporosis. Pediatr Endocrinol Rev 6 (Suppl 1): 81-85, 2008.

6. Jian J, Pelle E and Huang X: Iron and menopause: Does increased iron affect the health of postmenopausal women? Antioxid Redox Signal 11: 2939-2943, 2009.

7. Tsay J, Yang Z, Ross FP, et al: Bone loss caused by iron overload in a murine model: Importance of oxidative stress. Blood 116: 2582-2589, 2010.

8. de Vernejoul MC, Pointillart A, Golenzer CC, et al: Effects of iron overload on bone remodeling in pigs. Am J Pathol 116: 377-384, 1984.

9. Adams PC and Chakrabarti S: Genotypic/phenotypic correlations in genetic hemochromatosis: Evolution of diagnostic criteria. Gastroenterology 114: 319-323, 1998.

10. Zacharski LR, Ornstein DL, Woloshin S and Schwartz LM: Association of age, sex, and race with body iron stores in adults: Analysis of NHANES III data. Am Heart J 140: 98-104, 2000.

11. Huang X, Xu Y and Partridge NC: Dancing with sex hormones, could iron contribute to the gender difference in osteoporosis? Bone 55: 458-460, 2013.

12. Xu YJ, Sirois P and Li K: Iron overload plays a unique role in osteoporosis. Blood (E-letter) http://www.bloodjournal.org/cont ent/116/14/2582.e-letters\#iron-overload-plays-a-unique-role-i n-osteoporosis. Accessed May 6, 2015.

13. Kim BJ, Ahn SH, Bae SJ, et al: Iron overload accelerates bone loss in healthy postmenopausal women and middle-aged men: A 3-year retrospective longitudinal study. J Bone Miner Res 27: 2279-2290, 2012

14. Kim BJ, Lee SH, Koh JM and Kim GS: The association between higher serum ferritin level and lower bone mineral density is prominent in women $\geq 45$ years of age (KNHANES 2008-2010). Osteoporos Int 24: 2627-2637, 2013.

15. Clark SF: Iron deficiency anemia. Nutr Clin Pract 23: 128-141, 2008. 
16. Zimmermann MB and Hurrell RF: Nutritional iron deficiency. Lancet 370: 511-520, 2007.

17. Yang Q, Jian J, Katz S, Abramson SB and Huang X: 17 beta-Estradiol inhibits iron hormone hepcidin through an estrogen responsive element half-site. Endocrinology 153 : 3170-3178, 2012

18. Hou Y, Zhang S, Wang L, et al: Estrogen regulates iron homeostasis through governing hepatic hepcidin expression via an estrogen response element. Gene 511: 398-403, 2012.

19. Isomura H, Fujie K, Shibata K, et al: Bone metabolism and oxidative stress in postmenopausal rats with iron overload. Toxicology 197: 93-100, 2004.

20. Kalinowski DS and Richardson DR: The evolution of iron chelators for the treatment of iron overload disease and cancer. Pharmacol Rev 57: 547-583, 2005.

21. Bennett CN, Longo KA, Wright WS, et al: Regulation of osteoblastogenesis and bone mass by Wnt10b. Proc Natl Acad Sci USA 102: 3324-3329, 2005.

22. Almeida M, Han L, Martin-Millan M, O'Brien CA and Manolagas SC: Oxidative stress antagonizes Wnt signaling in osteoblast precursors by diverting beta-catenin from $\mathrm{T}$ cell factorto forkhead box O-mediated transcription. J Biol Chem 282 27298-27305, 2007

23. Jia P, Xu YJ, Zhang ZL, et al: Ferric ion could facilitate osteoclast differentiation and bone resorption through the production of reactive oxygen species. J Orthop Res 30: 1843-1852, 2012.

24. Liu G, Men P, Kenner GH and Miller SC: Age-associated iron accumulation in bone: implications for postmenopausal osteoporosis and a new target for prevention and treatment by chelation. Biometals 19: 245-251, 2006.

25. Liu G, Men P, Kenner GH and Miller SC: Therapeutic effects of an oral chelator targeting skeletal tissue damage in experimental postmenopausal osteoporosis in rats. Hemoglobin 32: 181-190, 2008.

26. Maggio A, Filosa A, Vitrano A, et al: Iron chelation therapy in thalassemia major: A systematic review with meta-analyses of 1520 patients included on randomized clinical trials. Blood Cells Mol Dis 47: 166-175, 2011.

27. Fabio G, Minonzio F, Delbini P, Bianchi A and Cappellini MD Reversal of cardiac complications by deferiprone and deferoxamine combination therapy in a patient affected by a severe type of juvenile hemochromatosis ( $\mathrm{JH})$. Blood 109: 362-364 2007.

28. Kalpatthi R, Peters B, Kane I, et al: Safety and efficacy of high dose intravenous desferrioxamine for reduction of iron overload in sickle cell disease. Pediatr Blood Cancer 55: 1338-1342, 2010.

29. Ishii KA, Fumoto T, Iwai K, et al: Coordination of PGC-1beta and iron uptake in mitochondrial biogenesis and osteoclast activation. Nat Med 15: 259-266, 2009.

30. Ganz T: Hepcidin, a key regulator of iron metabolism and mediator of anemia of inflammation. Blood 102: 783-788, 2003.

31. Donovan A, Brownlie A, Zhou Y, et al: Positional cloning of zebrafish ferroportin1 identifies a conserved vertebrate iron exporter. Nature 403: 776-781, 2000.

32. Nemeth E, Tuttle MS, Powelson J, et al: Hepcidin regulates cellular iron efflux by binding to ferroportin and inducing its internalization. Science 306: 2090-2093, 2004.

33. Lesbordes-Brion JC, Viatte L, Bennoun M, et al: Targeted disruption of the hepcidin 1 gene results in severe hemochromatosis. Blood 108: 1402-1405, 2006.

34. Nicolas G, Bennoun M, Devaux I, et al: Lack of hepcidin gene expression and severe tissue iron overload in upstream stimulatory factor 2 (USF2) knockout mice. Proc Natl Acad Sci USA 98: 8780-8785, 2001.

35. Hadziahmetovic M, Song Y, Ponnuru P, et al: Age-dependent retinal iron accumulation and degeneration in hepcidin knockout mice. Invest Ophthalmol Vis Sci 52: 109-118, 2011.

36. Roetto A, Papanikolaou G, Politou M, et al: Mutant antimicrobial peptide hepcidin is associated with severe juvenile hemochromatosis. Nat Genet 33: 21-22, 2003.

37. Ahmad KA, Ahmann JR, Migas MC, et al: Decreased liver hepcidin expression in the Hfe knockout mouse. Blood Cells Mol Dis 29: 361-366, 2002.
38. Guggenbuhl P, Fergelot P, Doyard M, et al: Bone status in a mouse model of genetic hemochromatosis. Osteoporos Int 22: 2313-2319, 2011

39. Nicolas G, Viatte L, Lou DQ, et al: Constitutive hepcidin expression prevents iron overload in a mouse model of hemochromatosis. Nat Genet 34: 97-101, 2003.

40. Moran-Jimenez MJ, Mendez M, Santiago B, et al: Hepcidin treatment in $\mathrm{Hfe}-/-$ mice diminishes plasma iron without affecting erythropoiesis. Eur J Clin Invest 40: 511-517, 2010.

41. Gardenghi S, Ramos P, Marongiu MF, et al: Hepcidin as a therapeutic tool to limit iron overload and improve anemia in beta-thalassemic mice. J Clin Invest 120: 4466-4477, 2010.

42. Andrews NC: Closing the iron gate. N Engl J Med 366: 376-377, 2012.

43. Ganz T and Nemeth E: The hepcidin-ferroportin system as a therapeutic target in anemias and iron overload disorders. Hematology Am Soc Hematol Educ Program 2011: 538-542, 2011.

44. Li GF, Pan YZ, Sirois P, Li K and Xu YJ: Iron homeostasis in osteoporosis and its clinical implications. Osteoporos Int 23: 2403-2408, 2012

45. Ma Y, Xu YJ, Wang AD, Yu C, Wang B, Zhang P and Zhang ZD: A preliminary report of expression of hepcidin gene in SD rats osteoporosis model. Su Zhou Da Xue Zue Bao 26: 367-369, 2006 (In Chinese).

46. Zhang $\mathrm{P}, \mathrm{Xu}$ YJ, Zhao DY, et al: Increased intracellular iron and mineralization of cultured hFOB 1.19 cells following hepcidin activation through ferroportin-1. Saudi Med J 31: 1303-1308, 2010.

47. Li GF, Xu YJ, He YF, et al: Effect of hepcidin on intracellular calcium in human osteoblasts. Mol Cell Biochem 366: 169-174, 2012.

48. Xu Y, Li G, Du B, et al: Hepcidin increases intracellular $\mathrm{Ca}^{2+}$ of osteoblast hFOB1.19 through L-type $\mathrm{Ca}^{2+}$ channels. Regul Pept 172: 58-61, 2011

49. Xi Huang: Treatment of osteoporosis in peri- and post-menopausal women with hepcidin. US Patent 0,204,122. Filed February 11, 2010; issued August 12, 2010.

50. De Domenico I, Lo E, Ward DM and Kaplan J: Hepcidin-induced internalization of ferroportin requires binding and cooperative interaction with Jak2. Proc Natl Acad Sci USA 106: 3800-3805, 2009.

51. De Domenico I, Zhang TY, Koening CL, et al: Hepcidin mediates transcriptional changes that modulate acute cytokine-induced inflammatory responses in mice. J Clin Invest 120: 2395-2405, 2010.

52. Bellido T, Borba VZ, Roberson P, Manolagas SC: Activation of the Janus kinase/STAT (signal transducer and activator of transcription) signal transduction pathway by interleukin-6-type cytokines promotes osteoblast differentiation. Endocrinology 138 3666-3676, 1997.

53. Nishimura R, Moriyama K, Yasukawa K, Mundy GR and Yoneda T: Combination of interleukin-6 and soluble interleukin-6 receptors induces differentiation and activation of JAK-STAT and MAP kinase pathways in MG-63 human osteoblastic cells. J Bone Miner Res 13: 777-785, 1998.

54. Barhanpurkar AP, Gupta N, Srivastava RK, et al: IL-3 promotes osteoblast differentiation and bone formation in human mesenchymal stem cells. Biochem Biophys Res Commun 418: 669-675, 2012.

55. Xu Y, Zhang W, Zhang P, et al: Downregulation of ferroportin 1 expression in hFOB1.19 osteoblasts by hepcidin. Inflammation 35 : $1058-1061,2012$

56. Preza GC, Ruchala P, Pinon R, et al: Minihepcidins are rationally designed small peptides that mimic hepcidin activity in mice and may be useful for the treatment of iron overload. J Clin Invest 121: 4880-4888, 2011.

57. Katsumata S, Tsuboi R, Uehara M and Suzuki K: Dietary iron deficiency decreases serum osteocalcin concentration and bone mineral density in Rats. Biosci Biotechnol Biochem 70: 2547-2550, 2006

58. Maurer J, Harris MM, Stanford VA, et al: Dietary iron positively influences bone mineral density in postmenopausal women on hormone replacement therapy. J Nutr 135: 863-869, 2005. 